\title{
Crisis Behavior in Autism Spectrum Disorders: A Self-Organized Criticality Approach
}

\author{
Lucio Tonello $\mathbb{D}^{1},{ }^{1}$ Luca Giacobbi, ${ }^{2}$ Alberto Pettenon, ${ }^{2}$ Alessandro Scuotto, ${ }^{1}$ \\ Massimo Cocchi, ${ }^{3}$ Fabio Gabrielli, ${ }^{1}$ and Glenda Cappello $\mathbb{D}^{1}$ \\ ${ }^{1}$ LUDES (Higher Education Institution) Foundation, Smart City, Malta \\ 2"Ca'Leido" Autism Center, Altivole, Italy \\ ${ }^{3}$ University of Bologna, Bologna, Italy \\ Correspondence should be addressed to Lucio Tonello; luciotonello@gmail.com
}

Received 30 August 2017; Revised 19 December 2017; Accepted 8 January 2018; Published 31 January 2018

Academic Editor: David Arroyo

Copyright (C) 2018 Lucio Tonello et al. This is an open access article distributed under the Creative Commons Attribution License, which permits unrestricted use, distribution, and reproduction in any medium, provided the original work is properly cited.

\begin{abstract}
The Autism Spectrum Disorder (ASD) represents a set of life-long disorders. In particular, subjects with ASD can display momentary behaviors of acute agitation and aggressiveness called crisis behaviors. These events are problematic for the subject and care providers but little is known about their occurrence, namely, possible relations among intensity, frequency, and duration. A group of ASD subjects $(n=33)$ has been observed for 12 months reporting data on each crisis $(n=1137$ crises $)$. Statistical analysis did not find significant results, while the relation between crisis duration and frequency showed a good fit to a "power law" curve, suggesting the application of Self-Organized Criticality (SOC) model. The SOC is used to describe natural phenomena as earthquakes, bank failures of rivers, mass extinctions, and other systems where a type of "catastrophic events" is necessary to maintain a critical equilibrium. In a sense, subjects at risk of crisis behavior seem to fit the same model as seismic zones at risk of earthquakes. The employment of the same strategies, as those successfully developed for known SOC systems, could lead to important insights for ASD management. Moreover, the SOC model offers possible interpretations of crisis behavior dynamics suggesting that they are unpredictable and, in a sense, necessary.
\end{abstract}

\section{Introduction}

The Autism Spectrum Disorder (ASD) represents a set of lifelong disorders characterized by impairments in reciprocal social interactions, communication skills, and repetitive and stereotyped patterns of behavior, interests, and activities [1,2]. In addition to these core features, many other nonspecific behaviors, such as anxiety, depression, sleeping, and eating disorders, are quite common. It has been found that features of ASD are often accompanied by impairments in cognitive and adaptive functioning, learning styles, and attention skills $[3,4]$.

Notably, subjects with ASD can present momentary behaviors of acute agitation $[5,6]$. They can display temporary maladaptive or challenging behaviors such as oppositional or disturbing activities (e.g., echolalia and repetitive beating) as well as more severe behaviors such as dangerous self-injuries or aggressions. These multiple acute agitation forms (behavioral crisis) could be very different from one another in terms of intensity, duration, and frequency. They are often referred to, for short, as crisis behaviors [7]. Such crises seem hard to predict and once they occur, they can be difficult to manage by families, caregivers, educators, or any other possible care providers because subjects presenting with autism spectrum disorders are often not likely to respond positively to intervention strategies (e.g., verbal reassurance, coaxing, or explanations) that are usually performed $[7,8]$.

We believe that studying crisis behavior occurrence, hence investigating and understanding possible relations among intensity, frequency, and duration, could be very important. We wish to address the following questions. Is the kind of crisis (according to a type of intensity or severity) related to its duration or frequency? Are longer or shorter crises more frequent? Could it be previously known what the probability of their occurrence is? 
There is little information in the literature regarding these topics. Many authors have described trends related to frequency and severity changes (or intensity) in crisis behaviors for decades but what is reported appears not to be focused on searching for general rules or definite laws in an analytical way. Moreover, some results seem controversial [914]. It is noteworthy that crisis behavior has been described as the "tip of the iceberg" [15] suggesting that its underlying reason seems to be the result of a complex interplay of many different variables, almost impossible to identify in a clear way $[7,16]$. Besides searching for possible reasons, this framework led us to first focus on a statistical approach, considering crisis as events regardless of their causation, but in terms of their frequency and severity as previously shown. In fact, despite the literature being incomplete, pragmatically speaking, this information could be very useful and effective, for example, for crisis managing both from a single subject support point of view and from the administration perspective for a group on the autism spectrum (i.e., hospitals, health care centers, and clinics) [17].

In order to investigate frequency, intensity, and the duration of ASD crisis behavior, a group of ASD subjects has been observed for one year and each crisis behavior event has been recorded in a data base. Then, the data collected have been statistically described and studied through different analytical methods in order to reach the aim of the study, that is, to find possible relations between crisis behavior duration, intensity, and frequency in ASD subjects. The more interesting and promising result came not from conventional statistical methods (also performed and reported) but from the Self-Organized Criticality (SOC) model, a well-known analytical approach suggested by the complexity science $[18,19]$. We believe that this could lead to effective results for ASD crisis management both from an individual and from a community point of view. Moreover, it offers possible interesting interpretation of crisis behavior dynamics suggesting they could be considered, in a sense, necessary as well as unpredictable. To the best of our knowledge, no application of SOC to ASD has been reported in the scientific literature to date.

\section{Methods}

2.1. Aim of the Study. The aim of the study was the investigation of the occurrence of ASD crisis behavior, with reference to frequency, intensity, and duration and possible relations among them through a quantitative approach. In order to do that, crisis behavior data for a group of subjects $(n=$ 33) have been collected and recorded for one year. The data have then been analyzed using statistical and analytical tools and models. This information could be very useful and effective, for example, for crisis managing both from a single subject support point of view and within a group from the autism spectrum administration perspective (i.e., hospitals and health centers).

2.2. Data Collection and Variables Specifications. A group of young subjects $(n=33)$ with diagnosed ASD attending the "Ca'Leido" Autism Center (an ASD service facility recognized by the local agency of the Italian national health system) have been recruited. Basically, the subjects included in the study have all been the ASD subjects in the local health administration area who agreed to enter the Autism Center, who regularly attend it, and who consented to join the study (see the "Compliance with Ethical Standards" paragraph below). No other exclusion criteria have been applied. It should be noted that the Autism Center does not have any admission restrictions (e.g., a restriction based on any possible ASD severity scale or behavioral problems).

Subjects in the study have been observed for 1 year (starting from November 10, 2015) and each crisis behavior has been recorded in a data base (DB).

For every crisis, the DB reports its "duration," its "intensity," the date, and, of course, the identification code of the involved subject.

The "duration" has been reported with time units of 5 minutes, because both the start and the end of a single crisis are not precisely known but fuzzy. Hence, "duration $=1$ " means between 1 and 5 minutes, "duration $=2$ " means between 5 and 10 minutes, "duration $=3$ " means between 10 and 15 minutes, and so on.

The crisis definition and its "intensity" parameter have been chosen focusing on the social impairment side of a crisis. It has been defined according to the Functional Analysis Screening Tool (FAST) severity scale [20-22] in a three-level classification summarized below.

Level A: mild, disruptive but little risk to property or health

Level B: moderate, property damage or minor injury

Level C: severe, significant threat to health or safety

The level (A, B, or C) has been evaluated by $n=16$ expert health professionals properly trained and alternately working at the Autism Center.

2.3. Statistical Analysis. Data have been analyzed using Statistica 10 (StatSoft, Inc., STATISTICA data analysis software system, version 10. http://www.statsoft.com, 2011).

Data normality has been evaluated by the Shapiro-Wilk test. Data found were not normally distributed $(p>0.05)$; thus, nonparametric tests were needed and comparison has been performed with the Friedman test (significance level $p<$ 0.05).

The function estimation has been performed with Statistica 10 "Nonlinear Estimation Model" (user defined as $y=$ $\left.c x^{-t}\right)$, setting the Levenberg-Marquardt estimation method (convergence criterion $\left.=10^{-6}\right)$.

2.4. Limitation of the Study. The number of investigated subjects is limited to $n=33$. Moreover, they come from a definite health administration area and agreed to entering and attending the Autism Center facility: this could hide a geographic bias, as well as a selection bias. A greater multicentric population should be analyzed, recruiting subjects from different kinds of facilities to overcome such possible biases. 
Classification of crisis intensity (level A, B, or C) has been performed by expert health professionals. Even if they have been properly trained, and the classification criteria are clearly described in the scientific literature, it is always a subjective evaluation that could differ from one evaluator to another especially for borderline data. On the other hand, increasing the number of the evaluators $(n=16)$ could have reduced this kind of error.

The power law parameter estimation has been performed on the crisis behavior of all of the subjects (see "Results") in the data base. Such a result is consistent with the emergence of a power law for any single subject. Even if the formal analytical estimation of the data cannot be performed because of the small size of the single subject data set, each of their shapes (power law like) together with their overall (grouped) result allows us to reasonably assume the single subject power law distribution as one of the hypothesis to consider for a part of the "Discussion." Future work on a larger sample size would allow an analytical parameter estimation at the single subject level.

\section{Results}

The investigated subjects are $n=33$ ( 29 males and 4 females) in number. They range in age between 8 and 33 years with a medium value of $15.9 \pm 6.2(\mathrm{~m} \pm \mathrm{SD})$ years. The total number of recorded crisis events is 1137 (404 for level A, 321 for level $\mathrm{B}$, and 412 for level C).

The frequency per individual (number of crises per individual in the observed period) for level A, B, and C crises has been considered. As shown in Table 1, first row, no statistical differences $(p>0.05)$ emerged in crisis frequencies per individual among levels. This is consistent with a uniform distribution, thus suggesting looking at possible differences among levels in terms of total duration beside the mean crisis duration. Hence, the mean crisis duration per individual and the total duration per individual (i.e., the sum of the durations of all of the crises per individual) have been compared among levels of intensity. Once again, no significant differences appeared (see Table 1, second and third rows), so it has been shown that level $\mathrm{A}, \mathrm{B}$, and $\mathrm{C}$ crises seem to have similar duration. In short, observed crisis frequency and duration seem to be independent of the intensity (in terms of levels $\mathrm{A}, \mathrm{B}$, and $\mathrm{C})$.

Then, a different data analytical approach has been applied: for each level (A, B, and C), every single duration has been taken into account (see the "duration" definition in "Methods"). Hence, we have evaluated the following: the frequency of crisis with "duration 1," "duration 2," and so on (the maximum duration recorded has been "duration 100") for each of the three levels. We have found a value $(\mathrm{m} \pm \mathrm{SD})$ for level $\mathrm{A}=13.93 \pm 29.34$, level $\mathrm{B}=11.10 \pm 22.86$, and level $\mathrm{C}=$ $14.21 \pm 33.85$. Once again, no statistical significance appeared $(p>0.05)$ among levels. Hence, even this approach does not suggest a possible difference involving duration and intensity of crisis.

However, the curve shape emerging from the latter evaluation suggested a deeper investigation. The crisis duration versus frequency reminds a well-known relation. Namely, the

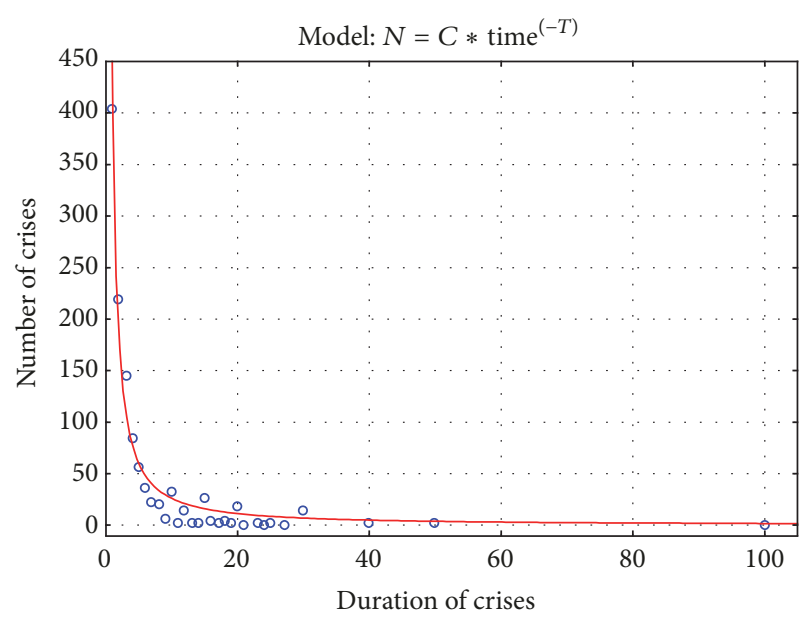

FIGURE 1: Blue circles represent the real data distribution of duration of crises versus number of crises in one year (scatter-plot graph), whose shape suggests a "power law" distribution. The analytical estimation of the power law distribution has been overimpressed (in red).

emerging distribution (see Figure 1) seems to fit the so-called "power law" [18], whose mathematical expression is given below:

$$
y=C x^{-T},
$$

where $y$ is the dependent variable; $x$ is the independent variable; $C$ and $T$ are constants depending on the single model.

Roughly speaking, the power law describes a relation in which a small number of crises have very long duration and, conversely, a very large number of crises have a short duration. Between these two extremes, the situation changes "regularly" according to a well-known function described by the so-called the power law curve, as shown in Figure 1 (red line).

Consequently, real data have been fitted using a nonlinear estimation model, according to the following distribution:

$$
N=C \text { Time }^{(-T)},
$$

where $N$ is number of crises in the observed period (frequency of crisis); time is crisis duration (unit of measurement is 5 minutes); $C, T$ are parameters to be found by software analysis.

The parameter estimation leads to the result reported in Table 2, with a proportion of variance accounted for PoV $=$ .97 and $R=.99$ (see Table 2). These numeric results suggest a very good fit to the observed data.

The resulting power law distribution plot is reported in Figure 1.

Dealing with a power law distribution, it is quite common to express the same data in a different way, achievable through a simple coordinates change, from linear to log-log for both the $x$ - and $y$-axis. This makes it clearer because, in the new coordinates system, the power law becomes linear so that the function $y=C x^{-T}$ becomes $Y=-T X+Z$ with $Y=\log (y)$, 
TABLE 1: Medium and SD value of crises number per individual in one year (i.e., frequency per individual), total duration per individual, and medium duration per individual, considered for each level of intensity (levels A, B, and C). In each cell, data are expressed as mean (SD).

\begin{tabular}{lcccc}
\hline & Level A & Level B & Level C & level of sig. \\
\hline Number of crises & $12.24(8.79)$ & $9.73(8.34)$ & $12.48(16.02)$ & n.s. \\
Total duration & $51.73(58.69)$ & $60.45(96.79)$ & $38.91(72.73)$ & n.s. \\
Mean duration & $4.08(3.77)$ & $4.59(5.00)$ & $2.30(1.53)$ & n.s. \\
\hline
\end{tabular}

TABLE 2: Power law parameters estimation.

\begin{tabular}{lccc}
\hline & Estimate & Standard E & $p$ value \\
\hline$C$ & 421.3647 & 14.41173 & 0.00 \\
$T$ & 1.2096 & 0.05256 & 0.00 \\
\hline
\end{tabular}

$X=\log (x)$, and $Z=\log (C)$. In our case, the $\log$-log equation becomes $Y=-1.2096 X+2.624658$.

\section{Discussion}

The evaluation of the crisis frequency and duration, both the total or medium value, has been shown to be similar (i.e., not statistically different) for levels A, B, and C, thus suggesting that frequency and duration are independent of the intensity. On the other hand, an interesting relation emerged: the duration of a crisis and its frequency are ruled by a power law distribution.

Interestingly, the power law is a fundamental element describing the well-known model called the "sand-pile model" [23]. To illustrate this, suppose we drop a grain of sand on a small table, then following a process of dropping grain after grain, a sand-pile forms, cone shaped. It will grow up in size until a definite, particular cone has been formed with a target shape (think of a particular cone height or side length or angle), which, once reached, cannot change any more, even adding sand grains again and again. In fact, adding new grains will not change the pile shape and dimension. This is possible because adding grain after grain occasionally causes landslides somewhere on the pile surface, in order to maintain the shape. Such landslides could be wider or smaller and could occur more or less frequently: the relation between their width (dimension) and their number (frequency) is not random but follows a power law distribution.

The sand-pile model is an example describing a selforganizing system in a state of equilibrium which is critical. Each grain drops and, suddenly, one of them causes, somewhere in the pile, a landslide of unknown dimension in order to maintain the critical shape. Notably, the system maintains its equilibrium not in a continuous or linear way but because of a sudden "catastrophic" event (e.g., landslides), happening at unknown times. The relation between the width and frequency of such events which follow, as stated before, a power law is known.

This critical equilibrium dynamics is an example of what is called the "Self-Organized Criticality" (SOC), a widely used model coming from the complexity science, employed to describe many different natural phenomena [18, 23]. SOC has been successfully applied in many fields for more than 20 years. One of the first was the statistics of earthquakes: the Earth's crust could be seen in a "critical state" and, occasionally, a catastrophic event (an earthquake) would happen in order to maintain its critical state of equilibrium [24]. The magnitude (a measure of width) of earthquakes and their number (frequency) have been shown to follow the power law. In the same way, bank failures of rivers [25], forest fires [26], mass extinctions [27], and many other systems have been successfully modeled by SOC and its power law. More recently, some applications have been shown within the field of neuroscience [28] as well as human behavior description $[29,30]$.

ASD crisis power law dynamics suggests their possible description using a SOC model. Subjects on the autism spectrum seem to fit the same dynamics as a system in critical equilibrium, in which, suddenly, a "catastrophic event" could occur: a crisis behavior could be modeled just as a landslide, an earthquake, a river bank failure, and so on, occurring in order to keep the system at a "critical equilibrium" (whatever it may mean for a subject with ASD).

Indeed, the finding of a power law does not necessarily imply that the observed system exhibits SOC: it is fundamental but is not the only element featuring this kind of model.

Marković and Gros [31] defined what could be considered a distinctive line of SOC models as "based on a very specific generating mechanism namely the separation of time scales between a fast internal dissipation [...] and a slow external driving." The ASD scientific literature [7, 15, 16, 32] suggests that crisis behavior (i.e., "fast internal dissipation") seems driven by many variables, named by Stark et al. [7] "daily life stressors" (i.e., "external variables"), interacting as a complex network: every day, stressor after stressor, add one after another, and interact, just as grains dropping and acting in sand-pile, slowly. Suddenly, a crisis behavior occurs, just as a sand-pile landslide, in a "separated" time scale.

Therefore, ASD crisis behavior dynamics seems to fit very well the SOC model. Moreover, Marković and Gros argue that a power law could describe two other lines of models as well, which, on the other hand, should be considered and, possibly, excluded.

The first is "based on external drives and internal dynamics competing on similar time scales and includes the coherent noise model": this should be trivially excluded because of its similar time scale feature, not present in ASD crisis behavior, as just illustrated.

The second one "proposes a noncritical self-organizing state, being guided by an optimization principle, such as the concept of highly optimized tolerance" (HOT). Carlson and Doyle [33] described the HOT model in which power 
law distributions can manifest themselves in systems with heterogeneous structures designed to operate optimally in uncertain environments without requiring critical dynamics. In the Marković and Gros [31] words, "the main difference between the SOC and the HOT mechanism is their explanation of large, possibly catastrophic events. [...] As for HOT, large events are caused by a design which favors small, frequent losses, having rather predictable statistics, over large losses resulting from rare perturbations" (HOT shows high robustness and resilience to "known" system failures and, on the other hand, high sensitivity to design flaws and unanticipated perturbations). Well, in ASD subject daily life stressors occur and interact with each other. A crisis behavior is, as previously reported, what Mesibov et al. [15] refers to as the "tip of the iceberg" in order to explain the underlying stressors' interplay. It seems not to be a system where different perturbations directly lead to different effects, according to their frequency of occurrence or probability, as the HOT one which, hence, should be excluded.

Hence, the SOC model can be reasonably argued to be the proper one.

Now, as the ASD crisis dynamics seem to fit it, we could hence suggest the employment of the same strategies, effectively and successfully developed for SOC systems [26].

As an example, let us go back to the earthquake example. One practical application of its SOC modeling is how the frequency of small and medium magnitude earthquakes in some areas can be used to quantify the risk of large ones: collecting data of small and medium earthquakes over a fixed area can define its own particular power law so that its risk of big magnitude earthquakes can be inferred. In other words, finding the parameters of the power law equations for each definite area (just using small and medium earthquakes data), we can describe a profile of its particular level of seismicity, thus ascribing its probability of big catastrophic events.

A subject on the autism spectrum could be modeled in the same way as a seismic zone, in which suddenly an "earthquake" (a crisis behavior) can occur. Collecting data on his/her crisis could easily lead to define his/her own particular power law equation, thus defining a particular "crisis" profile and risk. For example, as for an earthquake, its profile curve allowed the derivation of a probability of long duration crisis, whose risk of occurrence could be important, for example, because they are not always so easy to manage [5]. Basically, such a power law curve ascribed to a subject with ASD is a sort of crisis reference, just as the seismicity level of an earthquake prone area.

In general, the power law of subjects with ASD could be viewed (as in other SOC models) as a crisis profile. In fact, more clearly in its linear form (i.e., after the log$\log$ coordinates change), it can show a lot of information in an even easier way. Its slope, the area under the curve and all of the other mathematical values could give a lot of important information, which can be useful for real life effective applications. For example, in order to study the outcome (the effect) of a strategy or a treatment (behavioral as well as pharmacological or mixed): collecting data on frequency and duration of a subject crisis before and after the treatment allows us to build individual power law curves, hence his/her crisis profile before and after, which could be meaningfully compared. Changes in the equation parameters could be an objective measure, easy to find, easy to view, and easy to understand regarding the effect of a treatment, just as it could happen in other SOC modeled phenomena (e.g., just as interventions for fire prevention in a forest could change its fire power law distribution). Thus, knowing the crisis behavior risk profile could be helpful for managing both from a single subject support point of view as well from the administration perspective (i.e., hospitals, health care centers, and clinics) for a group with ASD.

Interestingly, the application of the SOC models to ASD, besides the description of empirical observations, suggests possible conceptual interpretations about the dynamics of the crisis behavior characteristics of this condition.

As previously seen, the SOC can describe natural phenomena in a "critical equilibrium" maintained through catastrophic events occurring, necessarily, according to a power law distribution. For example, a sand-pile maintains its cone shape through landslides and the Earth's crust maintains its equilibrium through earthquakes, obeying their power laws. Beyond the particular nature of any phenomenon described by the SOC model in its critical equilibrium, its catastrophic events must occur following the power law. In the same way, ASD crisis behaviors are necessary in order to obey the power law hence to maintain the "critical equilibrium." It must be pointed out that such equilibrium has to be understood as a "mathematical" concept, namely, within the theoretical framework given by the SOC model only, without any pathophysiological inference on the ASD subjects. The SOC model asserts, at a descriptive level, that crisis behaviors must fit the power law. In this sense, crisis behaviors are necessary (e.g., a "long" crisis must occur as it must fit the power law).

Secondly, according to the SOC, the occurrence of a particular "catastrophic event" is unpredictable. In a sand-pile, dropping grain after grain will cause a landslide occasionally, but which particular grain will cause it remains unknown. In the same way, even if the reasons for earthquakes are known, they cannot be foreseen and a particular trigger cannot be predicted. Hence, according to the SOC model, even if the reason of a crisis behavior could be known in general, the particular triggering event could not be predicted.

In summary, the SOC application to ASD, beyond proposing useful and effective real life application for ASD subject management, offers interesting possible interpretations of crisis behavior dynamics, suggesting they are necessary and unpredictable as other natural phenomena described by the same model.

\section{Conclusion}

The study of the duration, the intensity, and the frequency of ASD crisis in a group of subjects with ASD has been performed for one year.

No statistically significant result has been found relating duration and frequency with intensity. On the other hand, data observation led to the emergence of an interesting relation between the duration and the frequency of ASD crisis 
behavior. In fact, it has been shown that such a relation has a very good fit with a power law curve, leading us to approach the crisis dynamic using a SOC model.

Subjects with ASD seem to fit the same model as seismic zones and ASD crises as earthquakes. Time after time, a crisis (just as an earthquake) could occur in order to maintain a "critical" equilibrium, as described in the SOC model. This view allowed us to apply to ASD crisis management the same effective and successful strategies found for SOC systems over several decades of fruitful applications, for example, suggesting a long duration crisis risk.

Such an approach suggests possible effective real life applications, which could be useful in subjects on the autism spectrum management both from an individual and from a group point of view. Moreover, it offers interesting possible interpretations of crisis behavior dynamics, suggesting they are, in a sense, necessary and unpredictable.

The SOC model could open the door to a new approach to ASD crisis behavior so that it could be worthy of a deeper investigation.

\section{Ethical Approval}

All procedures were in accordance with the ethical standards of the "Ca'Leido" Autism Center Ethics Committee and with the 1964 Helsinki declaration and its later amendments.

\section{Consent}

Informed consent was obtained from all individual participants included in the study.

\section{Conflicts of Interest}

The authors declare that there are no conflicts of interest regarding the publication of this article.

\section{Acknowledgments}

The authors would like to thank all of the fantastic "fioi" of the "Ca'Leido" Autism Center, SONDA Società Cooperativa Sociale ONLUS, for their tireless, professional, and fundamental help. The authors acknowledge Dr. Silvia Scuotto for her linguistic help and Professor Jack A. Tuszynski for reviewing the manuscript language. Lucio Tonello would like to thank Professor Emanuele Spada, a lighthouse for him as well as for generations of students.

\section{References}

[1] American Psychiatric Association, Diagnostic and Statistical Manual of Mental Disorders, 5th (DSM-5), American Psychiatric Publishing, Washington, DC, USA, 2013.

[2] World Health Organization, The ICD-10 Classification of Mental and Behavioural Disorders: Clinical Descriptions and Diagnostic Guidelines, World Health Organization, Geneva, Switzerland, 2010.
[3] R. L. Gabriels and D. E. Hill, Growing Up with Autism: Working with School-Age Children and Adolescents, Guilford, New York, NY, USA, 2007.

[4] S. B. Mukherjee, "Autism spectrum disorders - diagnosis and management," The Indian Journal of Pediatrics, vol. 84, no. 4, pp. 307-314, 2017.

[5] J. J. McGonigle, A. Venkat, C. Beresford, T. P. Campbell, and R. L. Gabriels, "Management of Agitation in Individuals with Autism Spectrum Disorders in the Emergency Department," Child and Adolescent Psychiatric Clinics of North America, vol. 23, no. 1, pp. 83-95, 2014.

[6] V. Valkanova, F. Rhodes, and C. L. Allan, "Diagnosis and management of autism in adults," The Practitioner, vol. 257, no. 1761, pp. 13-16, 2013.

[7] K. H. Stark, J. C. Barnes, N. D. Young, and R. L. Gabriels, "Brief Report: Understanding Crisis Behaviors in Hospitalized Psychiatric Patients with Autism Spectrum Disorder-Iceberg Assessment Interview," Journal of Autism and Developmental Disorders, vol. 45, no. 11, pp. 3468-3474, 2015.

[8] N. Scarpinato, J. Bradley, K. Kurbjun, X. Bateman, B. Holtzer, and B. Ely, "Caring for the child with an autism spectrum disorder in the acute care setting," Journal for Specialists in Pediatric Nursing, vol. 15, no. 3, pp. 244-254, 2010.

[9] B. J. Freeman, B. Rahbar, E. R. Ritvo, T. L. Bice, A. Yokota, and R. Ritvo, "The stability of cognitive and behavioral parameters in autism: A twelve year prospective study," Journal of the American Academy of Child and Adolescent Psychiatry, vol. 30, no. 3, pp. 479-482, 1991.

[10] P. T. Shattuck, M. M. Seltzer, J. S. Greenberg et al., "Change in autism symptoms and maladaptive behaviors in adolescents and adults with an autism spectrum disorder," Journal of Autism and Developmental Disorders, vol. 37, no. 9, pp. 1735-1747, 2007.

[11] S. L. Hartley, D. M. Sikora, and R. McCoy, "Prevalence and risk factors of maladaptive behaviour in young children with autistic disorder," Journal of Intellectual Disability Research, vol. 52, no. 10, pp. 819-829, 2008.

[12] K. Gray, C. Keating, J. Taffe, A. Brereton, S. Einfeld, and B. Tonge, "Trajectory of behavior and emotional problems in autism," American Journal on Intellectual and Developmental Disabilities, vol. 117, no. 2, pp. 121-133, 2012.

[13] A. C. Woodman, L. E. Smith, J. S. Greenberg, and M. R. Mailick, "Change in Autism Symptoms and Maladaptive Behaviors in Adolescence and Adulthood: The Role of Positive Family Processes," Journal of Autism and Developmental Disorders, vol. 45, no. 1, pp. 111-126, 2014.

[14] K. E. Sanchack and C. A. Thomas, "Autism spectrum disorder: Primary care principles," American Family Physician, vol. 94, no. 12, pp. 972-979, 2016.

[15] G. B. Mesibov, V. Shea, and E. Schopler, The TEACCH Approach to Autism Spectrum Disorders, Plenum Press, New York, NY, USA, 2004.

[16] V. Guinchat, C. Cravero, L. Diaz et al., "Acute behavioral crises in psychiatric inpatients with autism spectrum disorder (ASD): recognition of concomitant medical or non-ASD psychiatric conditions predicts enhanced improvement," Research in Developmental Disabilities, vol. 38, pp. 242-255, 2015.

[17] H. R. Hall and J. Carolyn Graff, "Maladaptive behaviors of children with Autism: Parent support, stress, and coping," Comprehensive Child and Adolescent Nursing, vol. 35, no. 3-4, pp. 194-214, 2012.

[18] P. Bak, How Nature Works, Oxford University Press, Oxford, UK, 1997. 
[19] H. J. Jensen, Self-Organized Criticality: Emergent Complex Behavior in Physical and Biological Systems, vol. 10 of Cambridge Lecture Notes in Physics, Cambridge University Press, Cambridge, UK, 1998.

[20] B. A. Iwata and I. G. DeLeon, The Functional Analysis Screening Tool, The Florida Center on Self-Injury, University of Florida, Gainesville, FL, USA, 2005.

[21] B. A. Iwata, I. G. Deleon, and E. M. Roscoe, "Reliability and validity of the functional analysis screening tool.," Journal of Applied Behavior Analysis, vol. 46, no. 1, pp. 271-284, 2013.

[22] R. H. Zaja, L. Moore, D. J. Van Ingen, and J. Rojahn, "Psychometric comparison of the functional assessment instruments QABF, FACT and FAST for self-injurious, stereotypic and aggressive/destructive behaviour," Journal of Applied Research in Intellectual Disabilities, vol. 24, no. 1, pp. 18-28, 2011.

[23] P. Bak, C. Tang, and K. Wiesenfeld, "Self-organized criticality: An explanation of the 1/f noise," Physical Review Letters, vol. 59, no. 4, pp. 381-384, 1987.

[24] P. Bak and C. Tang, "Earthquakes as a self-organized critical phenomenon," Journal of Geophysical Research: Solid Earth, vol. 94, no. B11, pp. 15635-15637, 1989.

[25] M. Fonstad and W. A. Marcus, "Self-organized criticality in riverbank systems," Annals of the Association of American Geographers, vol. 93, no. 2, pp. 281-296, 2003.

[26] B. D. Malamud, G. Morein, and D. L. Turcotte, "Forest fires: An example of self-organized critical behavior," Science, vol. 281, no. 5384, pp. 1840-1842, 1998.

[27] T. Krink and R. Thomsen, "Self-organized criticality and mass extinction in evolutionary algorithms," in Proceedings of the 2001 Congress on Evolutionary Computation, vol. 2, pp. 11551161, Seoul, South Korea, 2001.

[28] A. Levina, J. M. Herrmann, and T. Geisel, "Dynamical synapses causing self-organized criticality in neural networks," Nature Physics, vol. 3, no. 12, pp. 857-860, 2007.

[29] E.-J. Wagenmakers, S. Farrell, and R. Ratcliff, "Human cognition and a pile of sand: A discussion on serial correlations and self-organized criticality," Journal of Experimental Psychology: General, vol. 134, no. 1, pp. 108-116, 2005.

[30] R. T. Ramos, R. B. Sassi, and J. R. C. Piqueira, "Self-organized criticality and the predictability of human behavior," New Ideas in Psychology, vol. 29, no. 1, pp. 38-48, 2011.

[31] D. Marković and C. Gros, "Power laws and self-organized criticality in theory and nature," Physics Reports, vol. 536, no. 2, pp. 41-74, 2014.

[32] C. A. Mazefsky, J. Herrington, M. Siegel et al., "The role of emotion regulation in autism spectrum disorder," Journal of the American Academy of Child and Adolescent Psychiatry, vol. 52, no. 7, pp. 679-688, 2013.

[33] J. M. Carlson and J. Doyle, "Highly optimized tolerance: A mechanism for power laws in designed systems," Physical Review E: Statistical, Nonlinear, and Soft Matter Physics, vol. 60, no. 2, pp. 1412-1427, 1999. 


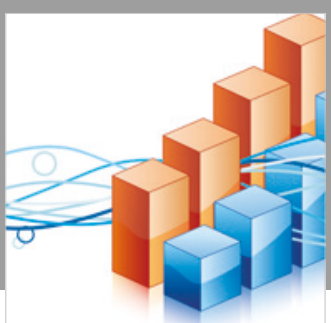

Advances in

Operations Research

\section{-n-m}
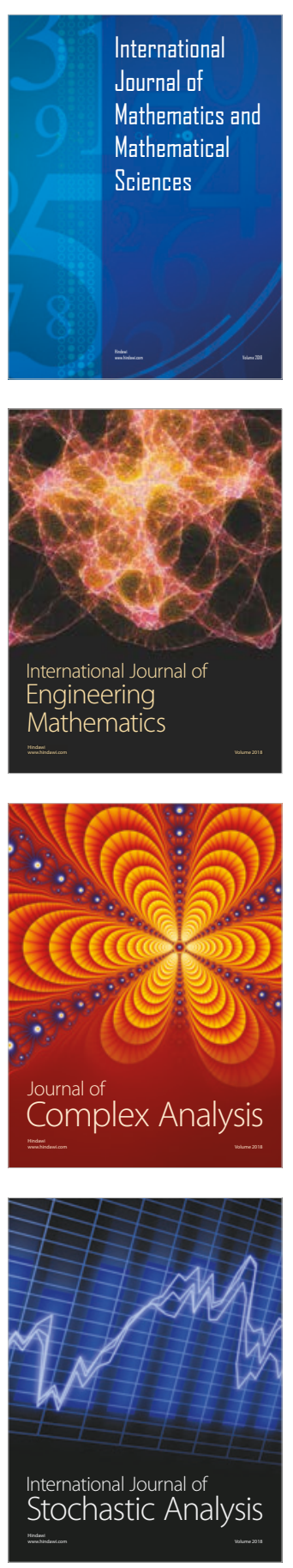
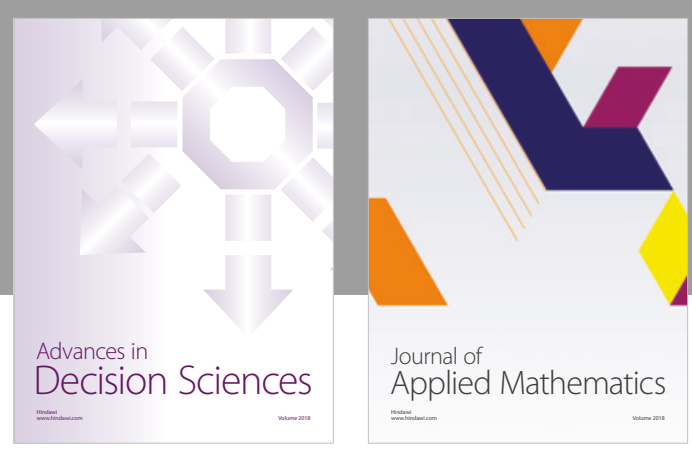

Journal of

Applied Mathematics
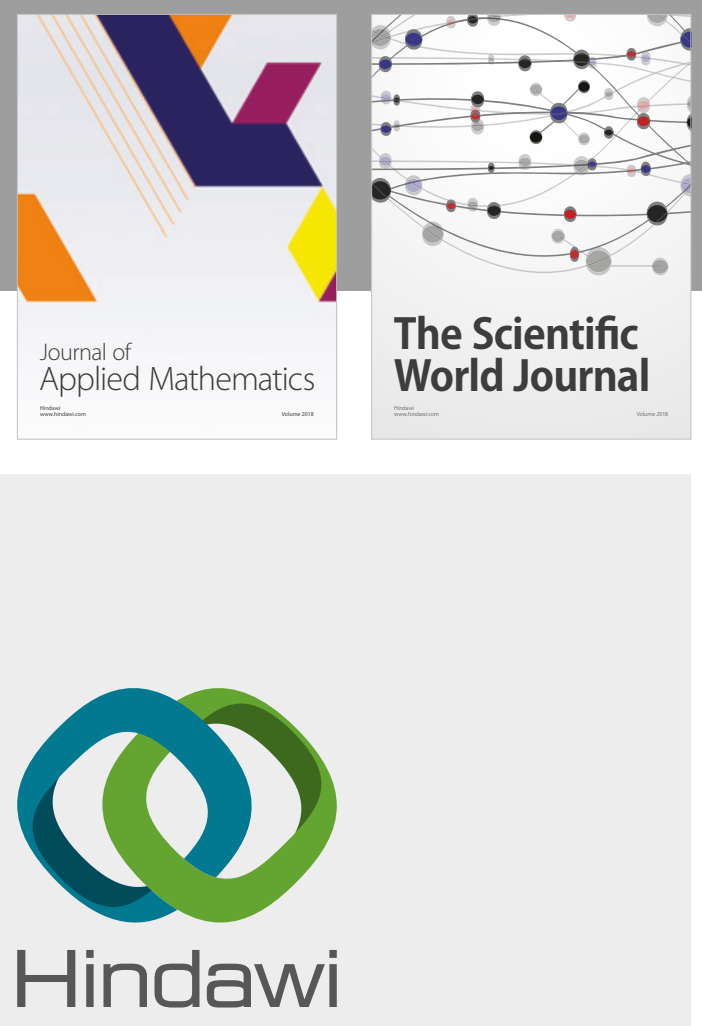

Submit your manuscripts at

www.hindawi.com

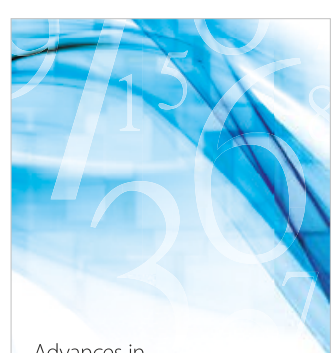

Advances in
Numerical Analysis
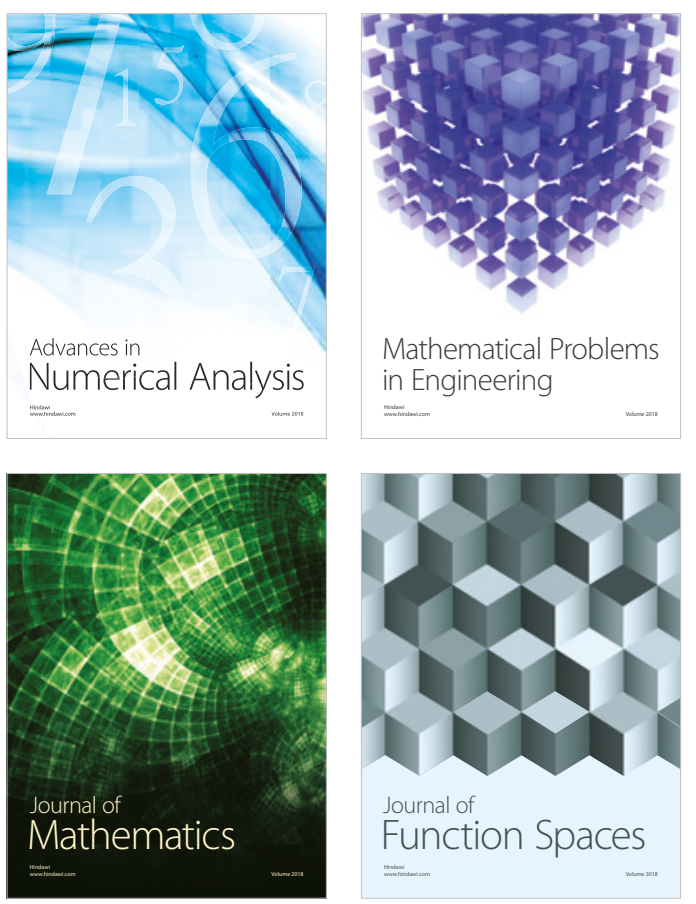

Mathematical Problems in Engineering

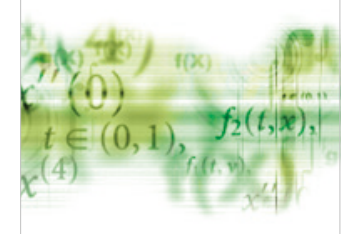

International Journal of

Differential Equations

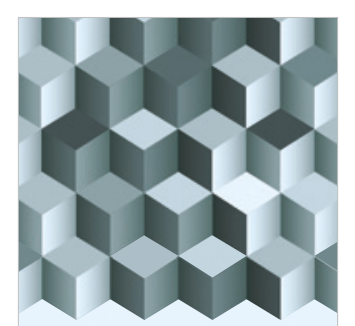

Journal of

Function Spaces
The Scientific

World Journal

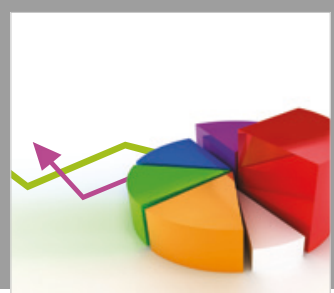

Journal of

Probability and Statistics
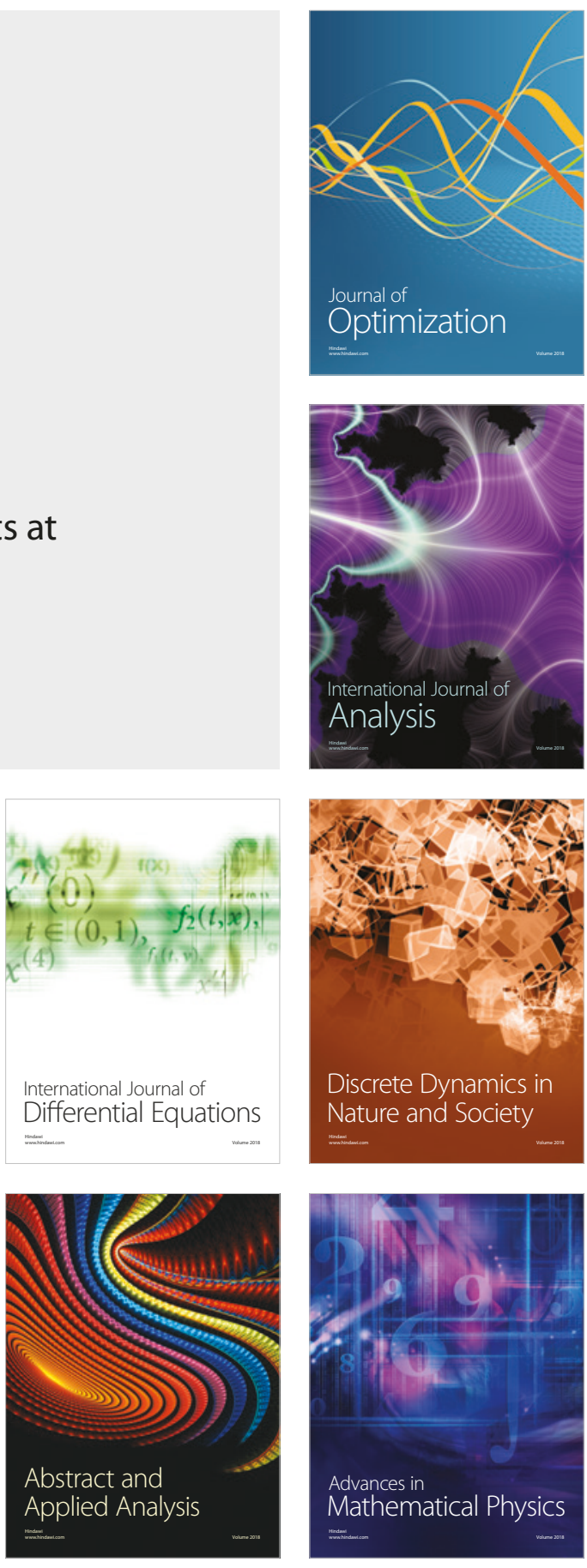